\title{
Midterm results of endovascular treatment of complicated acute type $B$ aortic dissection
}

\author{
Ali Khoynezhad, MD, ${ }^{\mathrm{a}}$ Carlos E. Donayre, MD, ${ }^{\mathrm{b}}$ Bassam O. Omari, MD, ${ }^{\mathrm{c}}$ George E. Kopchok, BS, ${ }^{\mathrm{b}}$ \\ Irwin Walot, $\mathrm{MD},{ }^{\mathrm{d}}$ and Rodney A. White, $\mathrm{MD}^{\mathrm{b}}$
}

Objectives: The operative mortality and morbidity of patients with complicated acute type B aortic dissection remain high. The endovascular approach has been proposed as a potential alternative. The purpose of this study is to review the contemporary outcome of patients undergoing endovascular treatment for complicated acute type B aortic dissection.

Methods: A retrospective analysis of 28 patients undergoing endovascular interventions for acute type B aortic dissection was performed. Kaplan-Meier survival analysis was used for statistical computation.

Results: Indications for emergency endografting were rupture in $4(14 \%)$ patients, severe lower body malperfusion in $8(29 \%)$ patients, visceral/renal malperfusion in $7(25 \%)$ patients, persistent chest pain despite proper antiimpulsive therapy in $5(18 \%)$ patients, uncontrollable hypertension in $1(4 \%)$ patient, and acute dilatation of false lumen with impending rupture in $3(11 \%)$ patients. Three $(11 \%)$ patients died early. Three patients died during follow-up of non-aorta-related causes. Overall survival was $82 \%$ and $78 \%$ at 1 and 5 years' follow-up, respectively. The aorta-related mortality was $10 \%$ for the entire follow-up period. Complete thrombosis of the false lumen in the thoracic aorta was achieved in $22(85 \%)$ members of the surviving cohort, and partial thrombosis was achieved in the remainder. The rate of treatment failure according to Stanford criteria was $18 \%$ at 5 years. Mean follow-up was 36 months, and follow-up was complete in $28(100 \%)$ patients.

Conclusions: Thoracic aortic endografting for complicated acute type B aortic dissection can be performed with a relatively low postoperative morbidity and mortality in experienced hands. The endovascular approach to lifethreatening complications of acute type B aortic dissection appears to have a favorable outcome in midterm follow-up.

Acute type $\mathrm{B}$ dissection is a dramatic and catastrophic condition. The majority of patients with uncomplicated disease can be treated conservatively with anti-impulsive and antihypertensive therapy. ${ }^{1}$ However, up to $20 \%$ of these patients can present with or later experience severe complications, such as rupture, impending rupture, or branch-vessel malperfusion. ${ }^{2}$ These challenging patients are usually triaged to surgical or endovascular management.

The surgical approach has been the standard of care for the treatment of complicated type B aortic dissection. However, management has been challenging, even in centers of excellence. ${ }^{3-5}$ The most recent results of surgical management of acute type B aortic dissection were presented by Bozinovski and Coselli. ${ }^{6}$ The in-hospital mortality was

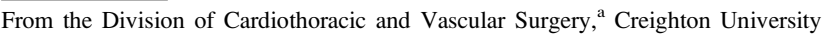
Medical Center, Omaha, Neb; and the Divisions of Vascular and Endovascular Surgery, ${ }^{\mathrm{b}}$ Cardiothoracic Surgery, ${ }^{\mathrm{c}}$ and Interventional Radiology, ${ }^{\mathrm{d}}$ Harbor-UCLA Medical Center, Torrance, Calif.

Read at the Thirty-fourth Annual Meeting of The Western Thoracic Surgical Association, Kona, Hawaii, June 25-28, 2008.

Received for publication June 26, 2008; revisions received April 4, 2009; accepted for publication April 22, 2009.

Address for reprints: Ali Khoynezhad, MD, PhD, Associate Professor of Cardiothoracic and Vascular Surgery, Director of Aortic and Endovascular Surgery, Creighton University Medical Center, 601 N 30th St, Suite 3700, Omaha, NE 68131 (E-mail: akhoy@creighton.edu).

J Thorac Cardiovasc Surg 2009;138:625-31

$0022-5223 / \$ 36.00$

Copyright (c) 2009 by The American Association for Thoracic Surgery

doi:10.1016/j.jtcvs.2009.04.044
}

$22.4 \%$, and almost two thirds of survivors experience at least 1 complication. ${ }^{6}$

For these reasons, thoracic endovascular aortic repair (TEVAR) of acute aortic dissection has gained increasing interest as an initial treatment option for patients with complicated acute type B aortic dissection. ${ }^{7-9}$ The goal of this therapy is to exclude the primary entry site, obliterate the false lumen, prevent aortic rupture, and relieve lower body malperfusion.

The aim of this study is to review the contemporary outcome of patients undergoing endovascular treatment for complicated acute type B aortic dissection.

\section{MATERIALS AND METHODS}

Seventy-two consecutive patients with type B aortic dissection underwent TEVAR between August 1999 and July 2007 at the Harbor-UCLA Medical Center and the University of Nebraska Medical Center. Twentyeight of these patients were admitted with complicated acute type B aortic dissection. The rest had chronic type B aortic dissection and were excluded from this study. Thirty procedures were performed in 19 (67.9\%) male and 9 female patients. After obtaining approval from the institutional review board, the patients were offered TEVAR through a single-institution investigator Investigational Device Exemption approved by the US Food and Drug Administration. All patients signed consent forms for the use of these investigational devices and agreed to participate in the surveillance protocols after deployment of the devices.

\section{Inclusion and Exclusion Criteria}

All patients had complicated acute type B aortic dissections and were candidates for TEVAR based on the adequacy of proximal and distal 


\section{Abbreviations and Acronyms \\ $\mathrm{CT}=$ computed tomographic \\ TEVAR $=$ thoracic endovascular aortic repair}

landing zones and access vessels. Furthermore, the patient's arterial anatomy must have met device-specific requirements to be a candidate for TEVAR. Other inclusion criteria were signing the informed consent form and agreeing to follow up in the institutional surveillance program.

The exclusion criteria included type A (or Retro-A) aortic dissection, chronic type B aortic dissection, penetrating ulcer or intramural hematoma of the descending thoracic aorta, an arterial anatomy unsuitable for TEVAR, connective tissue disorders, age less than 18 years, pregnancy, hypercoagulable disorder, and systemic infection. Advanced age or comorbidities per se were not part of the exclusion criteria.

\section{Definitions}

Aortic dissection was classified as type B according to the Stanford classification if the dissection did not involve the ascending aorta or aortic arch. The dissection was considered acute if the dissection presented within 2 weeks of the initial onset of symptoms. The term complicated dissection was defined as malperfusion syndromes, rupture, impending rupture, and persistent/unrelenting back pain or incontrollable hypertension despite maximal medical therapy.

Primary technical success was defined as complete exclusion of the primary tear site by the stent graft without procedural endoleak, death, or need for conversion to open repair. Treatment failure was defined according to the Stanford criteria as aortic rupture, device mechanical fault, reintervention, aorta-related death, or sudden or unexplained late death. ${ }^{10}$ Endoleaks were reported as defined by the reporting standard of Chaikof and colleagues. ${ }^{11}$

\section{Imaging/Endovascular Techniques}

Endovascular techniques used for TEVAR have been described in previous publications. ${ }^{12-15}$ All procedures were performed in the endovascular suite or operating room with C-arm fluoroscopy. Intravascular ultrasonographic analysis was used as adjunct imaging in every case to ensure location of the guidewire in the true lumen, assist with sizing of the stent graft, and evaluate the location of the primary intimal tear and the intraoperative end points of TEVAR (complete exclusion of the primary intimal tear and stagnation of blood flow in the false lumen). Continuous intraoperative transcranial Doppler monitoring was performed in the last 11 patients. The follow-up physical examination, contract computed tomographic (CT) scanning, and laboratory work were performed according to the institutional surveillance protocol at 1, 6, and 12 months and yearly thereafter. The protocol for postoperative imaging includes a 3-phase multidetector CT angiogram. A noncontrast scan through the chest and abdomen is followed by contrast CT angiographic analysis with $100 \mathrm{~mL}$ of nonionic contract. A 2-minute delayed CT scan is performed to increase the detection rate for endoleaks.

\section{Statistical Analysis}

Patient data, including demographics, risk factors, clinical symptoms, procedural details, CT scans, angiograms, postoperative complications, secondary interventions, and mortality, were collected in a retrospective manner by means of chart review and by means of review of prospective and concurrent US Food and Drug Administration reports of the respective Investigational Device Exemption protocols. All data were entered into an electronic database. Statistical analysis was performed with the SPSS 13.0 for Windows statistical software package (SPSS, Inc, Chicago, Ill). The actuarial survival rate was computed according to the Kaplan-Meier log-rank method. The statistical analysis underwent a mathematic review by a biostatistician.

\section{RESULTS}

Preoperative characteristics and outcomes of the patients undergoing TEVAR for complicated type B aortic dissection are summarized in Table 1. Indications for emergency TEVAR were rupture in $4(14 \%)$ patients, severe lower body malperfusion in $8(29 \%)$ patients, visceral/renal malperfusion in 7 $(25 \%)$ patients, persistent chest pain despite proper antiimpulsive therapy in $5(18 \%)$ patients, uncontrollable hypertension in $1(4 \%)$ patient, and acute dilatation of false lumen with impending rupture in $3(11 \%)$ patients. The latter was characterized by an enlarged aortic diameter in the dissected region with evidence of hemothorax and no active contrast extravasations in the $\mathrm{CT}$ imaging. Twenty-five patients underwent endovascular repair with the Talent device (Medtronic, Minneapolis, Minn). The TAG device (Gore, Flagstaff, Ariz) was deployed in the remaining 5 patients.

Adjunct procedures were necessary in 3 patients with static or combined dynamic and static obstruction of the branched vessels. Fenestration of the distal aortic dissection membrane was performed in a 63-year-old man with subsequent relief of visceral and limb ischemia. The postoperative course was complicated by multiorgan dysfunction, adult respiratory distress syndrome, and deterioration of renal function (without requiring hemodialysis). However, he fully recovered and has done well in more than 7 years of follow-up. A 58-year-old man with altered mental status and paraplegia on presentation was treated with fenestration of the perirenal aorta obviating the hemodialysis. Although his renal function normalized within the next 7 days, he remains paraparetic in the follow-up period. A 72-year-old patient with gut ischemia and severe lactic acidosis underwent salvage fenestration of an infradiaphragmatic aorta and antegrade stenting of the superior mesenteric artery. Retrograde access to the superior mesenteric artery was not achieved because of static obstruction of the visceral vessels. He died of multiorgan dysfunction syndrome on postoperative day 2 .

\section{Early and Late Mortality}

There were no intraprocedural deaths. The in-hospital, early, and aorta-related mortality was $10.7 \%$ (3/28), respectively. Two patients died of visceral ischemia and multiorgan dysfunction syndrome on postoperative days 2 and 3 . A third patient died of retrograde type A dissection on postoperative day 8 . Three additional patients died in the followup period (pneumonia in 2 patients and malignancy in the third patient). Overall survival according to Kaplan-Meier analysis was $82 \%$ and $78 \%$ at 1 and 5 years' follow-up, respectively (Figure 1). The mean follow-up was 36 months (standard deviation, \pm 27 months; range, 1-88 months), and follow-up was complete in all patients. 
TABLE 1. Perioperative demographics of the patients

\begin{tabular}{lc}
\hline \multicolumn{1}{c}{ Characteristics } & All patients (n= 28) \\
\hline Age, y (median) & $62 \pm 15($ range, 35-88) \\
Preoperative neurologic deficit (3 strokes & $4(14 \%)$ \\
$\quad$ and 1 paraplegia) & $5(18 \%)$ \\
s/p aortic surgery (4 open abdominal aortic & \\
$\quad$ aneurysm repairs and 1 ascending aortic & \\
$\quad$ replacement) & $1.5 \pm 1.4$ \\
Preoperative creatinine (mg/dL) & $49(26 \%)$ \\
Coronary artery disease & $1(3 \%)$ \\
Preoperative myocardial infarction & $4(14 \%)$ \\
Congestive heart failure & $7(25 \%)$ \\
Chronic obstructive pulmonary disease & $3(11 \%)$ \\
Peripheral vascular disease & $1(3.3 \%)$ \\
Postoperative stroke & 0 \\
Postoperative paraplegia & $3(10 \%)$ \\
Need for iliac conduit & $4 \pm 5.3$ \\
ICU stay, d (median) & $8 \pm 8.5$ \\
Hospital stay, d (median) & $3(10 \%)$ \\
Acute renal failure (creatinine $\geq 1.8 \mathrm{mg} / \mathrm{dL})$ & $122 \pm 70$ \\
Contrast injected (mL) & $10.9 \pm 7.7$ \\
Fluoroscopic time (min) & $152 \pm 165$ \\
Median operative time (min) &
\end{tabular}

\section{Technical Failures}

Primary technical success was achieved in $27(90 \%)$ of 30 patients. In 3 patients there was a small type Ia endoleak at the end of the procedure. Although the endoleak subsided in 1 patient after reversal of heparin and on subsequent follow-up imaging, the second patient required a reintervention to improve the apposition of the stent graft to the proximal landing zone. A third patient died of sudden death on postoperative day 8 (as described above). The autopsy examination revealed retrograde type A aortic dissection, which was not present on the day of the operation. No patient underwent conversion to open repair. There were no aortic ruptures in the follow-up period. The rate of treatment failure according to Stanford criteria was 5 $(18 \%)$ of 28 at 5 years.

During the follow-up period, there were 3 (aforementioned) early endoleaks $(10 \%)$, and a total of $5(20 \%)$ of 25 late endoleaks (endoleaks after 30 days): $80 \%(4 / 5)$ were type Ib endoleaks, and $5 \%(1 / 5)$ were type III endoleaks. Two patients required secondary interventions. A 40-year-old woman had proximal maldeployment and kinking of the stent graft. As mentioned above, she underwent re-expansion of the proximal neck with a Palmaz stent on postoperative day 2. A 63-year-old man continued to have visceral malperfusion requiring additional stent grafting of secondary intimal tears in the descending thoracic aorta and fenestration. No further secondary interventions were performed to treat an endoleak in the follow-up period.

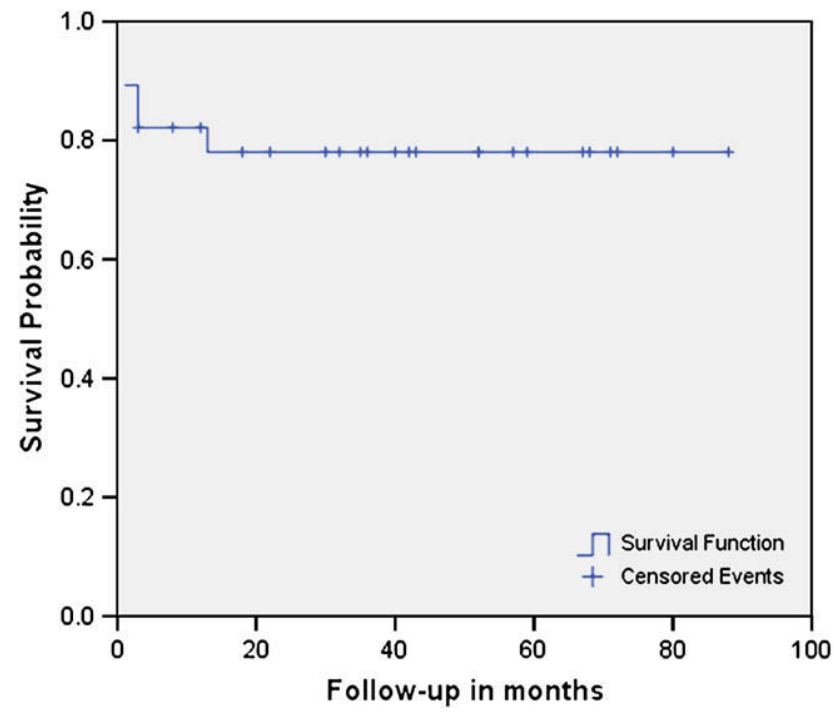

FIGURE 1. Kaplan-Meier survival curve. Tick marks indicate at least 1 patient censored because of length of follow-up.

\section{Patency of False Lumen}

Complete obliteration of the false lumen was achieved in $22(88 \%)$ patients in the follow-up period, and partial thrombosis was present in the remainder (Figure 2). In 3 patients with partial thrombosis, the false lumen was patent in the distal one third of the thoracic aorta and abdominal aorta in 2 patients, whereas 1 patient had evidence of false lumen perfusion in solely the abdominal aorta. On the latest imaging, the maximal aortic diameter and aortic volume increased in both patients with a persistent false lumen in the thoracoabdominal aorta.

\section{DISCUSSION}

Endovascular management of complicated acute type B dissection is complex and challenging. It has become an emerging alternative to open repair in many centers since its initial feasibility and safety was demonstrated by the Stanford group. ${ }^{16}$ The indications for endovascular therapy for complicated acute type B aortic dissection have yet to be determined. In the current era, the purest indications for TEVAR in the setting of acute type B aortic dissection are malperfusion and rupture. These critically ill patients have very high open surgical mortality, even in centers of excellence. ${ }^{3,6}$ Although validation studies comparing surgical therapy with TEVAR are missing, the endovascular approach has been used increasingly for these indications. ${ }^{17}$ The majority of patients in the current study $(85 \%)$ were treated for malperfusion rupture or impending rupture. Six other patients were treated for unrelenting chest/back pain or uncontrollable hypertension. These later indications are "softer calls" for TEVAR for acute type B aortic dissection. However, intractable pain is thought to be a symptom of progression of dissection, possible impending rupture, or malperfusion, necessitating endovascular intervention. . $^{6,10,17,18}$ 


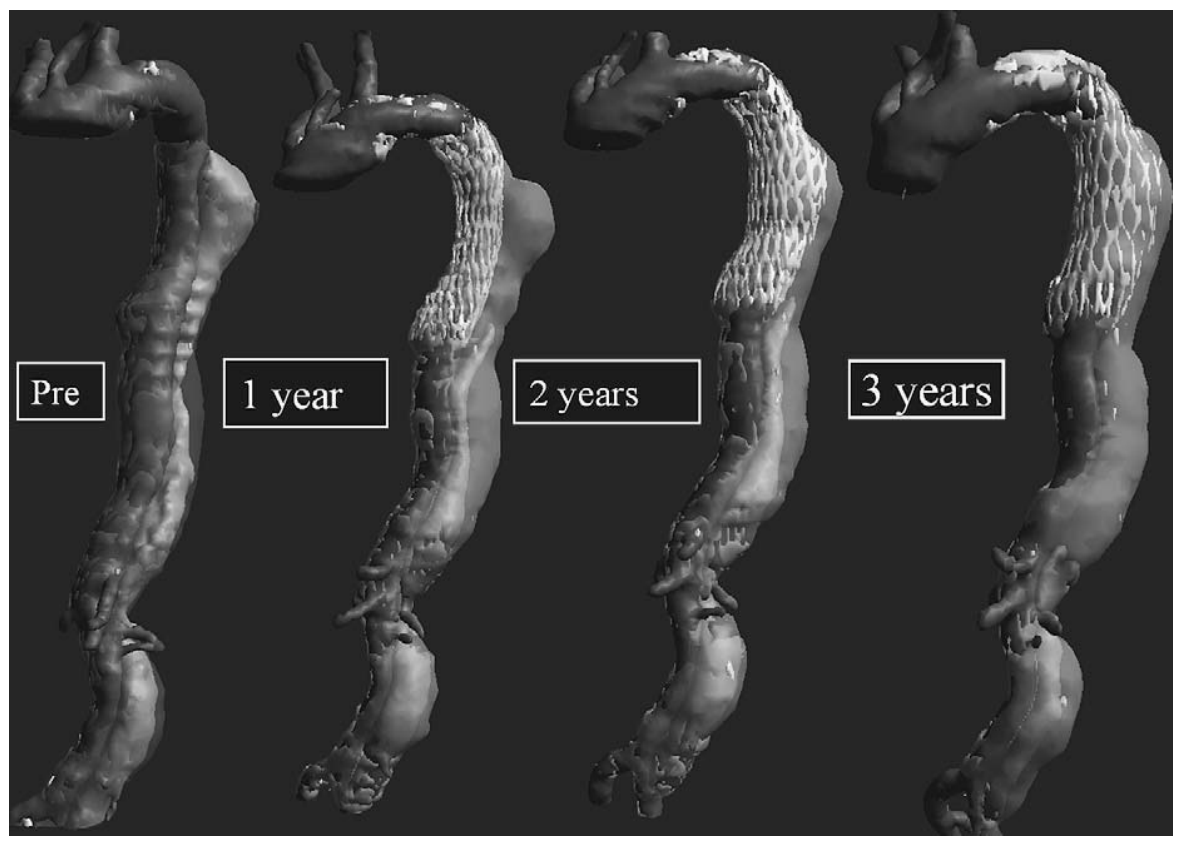

FIGURE 2. Incomplete reverse aortic remodeling of a complicated type B aortic dissection. The M2S (West Lebanon, NH) reconstruction of an axial computed tomographic scan demonstrates complete thrombosis of the false lumen of the proximal and mid descending thoracic aorta at 1 year's follow-up. The false lumen of the distal descending thoracic aorta takes another 2 years to completely clot because of multiple re-entry sites in the abdominal aorta. The false lumen in the abdominal aorta remains perfused at 3 years' follow-up.

Uncontrollable hypertension is a less common problem with modern antihypertensive and anti-impulsive strategies..$^{19,20}$ However, dissection into the renal arteries can cause poorly controlled or uncontrollable arterial hypertension because of an uncoupled renin-angiotensin-aldasterone system. Although these patients might not have increased creatinine levels and frank renal ischemia, many authors postulate surgical intervention or TEVAR to reduce the chance of rupture or early aneurysmal degeneration in the proximal descending thoracic aorta. ${ }^{6,10,19}$ As increased follow-up and experience has been gained for TEVAR in various patients with complicated acute type B aortic dissection, we hope to refine patient selection criteria and enhance physician judgment along with the evolution of more sophisticated, small-caliber, flexible, and pathology-specific TEVAR devices and deployment systems.

The aim of this study was to report the contemporary outcome of patients undergoing TEVAR for complicated acute type B aortic dissection. To the best of our knowledge, this series is the largest with complete 3-year average follow-up published in the literature. All patients underwent close and complete follow-up because of stringent study protocols and strict regulatory reasons. The results of this study are in concordance with those of the series reported originally by the Stanford group. ${ }^{10}$ Sixteen patients were treated with TEVAR for complicated type B aortic dissection (rupture, hemothorax, refractory chest pain, and/or severe visceral or lower limb ischemia) in this series. Early mortality was $23 \%$, with no late deaths. Based on the latest scan, 4
(25\%) patients had complete thrombosis of the false lumen, and $6(38 \%)$ patients had partial thrombosis. Aortic diameter increased in 1 patient. Actuarial survival was $73 \%$ at 1and 5-year intervals. The actuarial estimate of freedom from treatment failure was $67 \%$ at 5 years. ${ }^{10}$ A similar range of early survival, primary technical success, and treatment failure has been reported in the literature. ${ }^{17,21-24}$

Complete obliteration of the false lumen is one of the important goals in the follow-up of patients with acute type B aortic dissection. The European multicenter study by Erbel and coworkers ${ }^{25}$ showed that late outcome was better in patients with a thrombosed false lumen than in those with a patent false lumen. In the current study 22 of 25 early survivors had complete obliteration of the false lumen in the thoracoabdominal aorta. This high rate of aortic "reverse remodeling" is possible when the patient is treated early after development of the dissection flap. With progression of time, the dissection flap becomes more fibrosed, thickened, and matured, making it less compliant to TEVAR.

There are several limitations to the current study. It represents a retrospective analysis of prospectively collected data as part of investigational protocols at 2 institutions. There is a lack of a risk-adjusted control group undergoing open repair for similar aortic pathologies. A larger trial with a multicenter registry is recommended to substantiate the findings in this study and to establish long-term $(\geq 10$ years) durability and safety of the stent grafts in complicated acute type B aortic dissection. 
Furthermore, the authors have used first-generation stiffer stent grafts intended for treating aneurysms and have gone through a learning curve for this new technology. Some of the morbidities and mortalities encountered in the current series might be reduced/obviated based on our current experience. For instance, to reduce the possibility of retrograde type A aortic dissection, we do not balloon dilate the proximal landing zone and avoid oversizing by more than $15 \%$. Device sizing is performed based on the normal proximal aorta after the left common carotid artery. The primary end points of endovascular interventions are coverage of the primary entry site, relief of malperfusion (if present), and stagnation of blood in the false lumen. Intravascular ultrasonographic scanning has been instrumental in negotiation of the true lumen, accurate measurement of the dimensions of the proximal landing zone, visualization of (large) secondary entry sites, and evaluation for stagnation of blood in the false lumen after the obliteration of the primary entry site. In case of frank ruptures, impending ruptures, uncontrolled hypertension, and unrelenting pain, the entire aorta between the subclavian and celiac arteries is covered. The availability of a dissection-specific stent graft should improve our outcomes in cases of malperfusion; we would cover the primary (or large) secondary entry site and use a bare stent to expand the true lumen throughout the length of the descending thoracic aorta.

In brief, this series is (to the best of our knowledge) the largest cohort with the longest follow-up in the literature, demonstrating favorable early and midterm results for patients undergoing TEVAR for complicated acute type B aortic dissection. Although long-term durability of TEVAR for complicated acute type B aortic dissection is yet to be established, we conclude that TEVAR is the therapy of choice for patients with complicated acute type B aortic dissection and that a paradigm shift is warranted from open surgical treatment to TEVAR in these undesirable surgical candidates.

\section{References}

1. Khoynezhad A, Plestis KA. Managing emergency hypertension in aortic dissection and aortic aneurysm surgery. J Card Surg. 2006;21(suppl 1):S3-7.

2. Hagan PG, Nienaber CA, Isselbacher EM, Bruckman D, Karavite DJ, Russman PL, et al. The International Registry of Acute Aortic Dissection (IRAD): new insights into an old disease. JAMA. 2000;283:897-903.

3. Miller DC, Mitchell RS, Oyer PE, Stinson EB, Jamieson SW, Shumway NE. Independent determinants of operative mortality for patients with aortic dissections. Circulation. 1984;70:I153-64.

4. Reul GJ, Cooley DA, Hallman GL, Reddy SB. Kyger ER 3rd, Wukasch DC. Dissecting aneurysm of the descending aorta. Improved surgical results in 91 patients. Arch Surg. 1975;110:632-40.

5. Crawford ES, Svensson LG, Coselli JS, Safi HJ, Hess KR. Aortic dissection and dissecting aortic aneurysms. Ann Surg. 1988;208:254-73.

6. Bozinovski J, Coselli JS. Outcomes and survival in surgical treatment of descending thoracic aorta with acute dissection. Ann Thorac Surg. 2008;85:965-71.

7. Nienaber CA, Fattori R, Lund G, Dieckmann C, Wolf W, von Kodolitsch Y, et al. Nonsurgical reconstruction of thoracic aortic dissection by stent-graft placement. N Engl J Med. 1999;340:1539-45.

8. Dake MD, Kato N, Mitchell RS, Semba CP, Razavi MK, Shimono T, et al. Endovascular stent-graft placement for the treatment of acute aortic dissection. $N$ Engl J Med. 1999;340:1546-52.
9. Hansen CJ, Bui H, Donayre CE, Aziz I, Kim B, Kopchok G, et al. Complications of endovascular repair of high-risk and emergent descending thoracic aortic aneurysms and dissections. J Vasc Surg. 2004;40:228-34.

10. Svensson LG, Kouchoukos NT, Miller DC, Bavaria JE, Coselli JS, Curi MA et al. Expert consensus document on the treatment of descending thoracic aortic disease using endovascular stent-grafts. Ann Thorac Surg. 2008;85(suppl): S1-41.

11. Chaikof EL, Blankensteijn JD, Harris PL, White GH, Zarins CK, Bernhard VM, et al. Reporting standards for endovascular aortic aneurysm repair. $J$ Vasc Surg. 2002;35:1048-60.

12. Khoynezhad A, Donayre CE, Bui H, Kopchok GE, Walot I, White RA. Risk factors of neurologic deficit after thoracic aortic endografting. Ann Thorac Surg. 2007;83(suppl):S882-9.

13. Song TK, Donayre CE, Walot I, Kopchok GE, Litwinski RA, Lippmann M, et al Endograft exclusion of acute and chronic descending thoracic aortic dissections. $J$ Vasc Surg. 2006;43:247-58.

14. Khoynezhad A, Donayre CE, Smith J, Kopchok GE, Walot I, White RA. Risk factors for early and late mortality after thoracic endovascular aortic repair. J Thorac Cardiovasc Surg. 2008;135:1103-9. e1-4.

15. Khoynezhad A, Kruse MJ, Donayre CE, White RA. Use of transcranial Doppler ultrasound in endovascular repair of a type B aortic dissection. Ann Thorac Surg. 2008;86:289-91.

16. Dake MD, Kato N, Mitchell RS, Semba CP, Razavi MK, Shimono T, et al. Endovascular stent-graft placement for the treatment of acute aortic dissection. $N$ Engl J Med. 1999;340:1546-52.

17. Eggebrecht H, Lönn L, Herold U, Breuckmann F, Leyh R, Jakob HG, et al. Endovascular stent-graft placement for complications of acute type B aortic dissection. Curr Opin Cardiol. 2005;20:477-83.

18. Elefteriades JA, Griep R. Surgical procedures: a primer. In: Elefteriades JA, ed Acute aortic disease. New York: Informa Healthcare; 2007.p.251-68.

19. Khoynezhad A. Antihypertensive therapy in a patient with type B and de novo type A aortic dissection: case study. Crit Care Clin. 2007;23(suppl 1):29-38.

20. Khoynezhad A, Dobesh PP, Stacy Z, Jalali Z. The role of intravenous dihydropiridine calcium channel blockers in the perioperative management of patients undergoing coronary artery bypass grafting. Curr Vasc Pharmacol. 2008;3:186-94.

21. Steingruber IE, Chemelli A, Glodny B, Hugl B, Bonatti J, Hiemetzbeger R, et al Endovascular repair of acute type $\mathrm{B}$ aortic dissection: midterm results. J Endovasc Ther. 2008;15:150-60.

22. Tespili M, Banfi C, Valsecchi O, Aiazzi L, Ricucci C, Guagliumi G, et al. Endovascular treatment of thoracic aortic disease: mid-term follow-up. Catheter Cardiovasc Interv. 2007;70:595-601.

23. Bockler D, Schumacher H, Ganten M, von Tengg-Kobligk H, Schwarzbach M, Fink $\mathrm{C}$, et al. Complications after endovascular repair of acute symptomatic and chronic expanding Stanford type B aortic dissections. J Thorac Cardiovasc Surg. 2006;132:361-8

24. Schoder M, Czerny M, Cejna M, Rand T, Stadler A, Sodeck GH, et al. Endovascular repair of acute type B aortic dissection: long-term follow-up of true and false lumen diameter changes. Ann Thorac Surg. 2007;83:1059-66.

25. Erbel R, Oelert H, Meyer J, Puth M, Mohr-Katoly S, Hausmann D, et al. Effect of medical and surgical therapy on aortic dissection evaluated by transesophageal echocardiography. Implications for prognosis and therapy. The European Cooperative Study Group on Echocardiography. Circulation. 1993;87:1604-15.

\section{Discussion}

Dr D. Craig Miller (Stanford, Calif). Ali, this is very good. We have to be careful with you here because "alihi" in Hawaiian means "chief," and that is close to Ali.

I have no objections whatsoever to your talk this morning, which is a far cry from what I said last year in New Mexico.

Dr Khoynezhad. I remember that very well. (laughter)

Dr Miller. I bet you do, and if you keep coming back every year, some day we will make you an honorary member, just like Urschel and Walt Wolfe, so welcome back to the Western Thoracic Association.

I really do appreciate you bringing this material to our attention because I agree with you wholeheartedly that the best and most life- 
saving application for thoracic aortic stent grafts is going to be exactly these patients: patients with acute type B aortic dissection with complications, who are dying right under your eyes. It is probably the application that is being less applied certainly worldwide and maybe even in the United States. Therefore you have tackled the toughest patients, and I think you have shown some spectacular results of which you should be proud.

I really do agree with all your conclusions this year. I have a couple quibbles, and it would not be correct if I did not, right? You had very tight inclusion/exclusion criteria. I am wondering what happened and how many there were who were excluded from stent grafting because they were too sick, could not sign, or did not have the right anatomy. Do you have any idea what your internal control group was and how well they did?

Dr Khoynezhad. In regard to open operations?

Dr Miller. Whatever you did.

Dr Khoynezhad. We had 3 patients who required open operations in the follow-up period. The first patient was operated on at Creighton University, and 2 patients were operated on at HarborUCLA. In the latter 2 patients, we did not have adequate access to vessels. The size of the iliac arteries was not adequate for endovascular procedures. Both patients underwent open operations. The third patient had an emergency operation for a ruptured acute type $\mathrm{B}$ aortic dissection at Creighton University. I try to avoid doing those operations as much as I can. Having said that, exclusion criteria were severe acidosis, refusal of treatment, no vital signs, any type A dissections, retro-A dissections, chronic dissection (ie, 14 days after symptoms), and any intramural hematoma or aortic ulcers. Any connective tissue disorders were not treated with endovascular treatment either.

Dr Miller. That is fine. I was just getting to the ones who were too sick and dying. You were going to end up doing a warm autopsy one way or another.

Dr Khoynezhad. There are patients with type A or type B dissections who are not offered open or endovascular procedures. There are some patients who are too sick. They are severely acidotic, and they decide not to be treated at all, or the patient's family declines to do that. I do not have the number for those.

Dr Miller. I am not sure these can truly be called midterm results. You did not show us in your graft or in the manuscript how many were remaining at risk at 2, 3, 4, and 5 years and particularly how many of those had a current CT scan. I will bet you the numbers are small, less than a handful, out beyond 2 years, and therefore it might be more appropriate to term this article "early results."

Dr Khoynezhad. Your point is well taken.

Dr Miller. Can you give us some numbers remaining at risk at 2, 3 , or 4 years?

Dr Khoynezhad. We started performing endovascular treatment of complicated type B aortic dissection at Harbor-UCLA in 1999 after the initial publication from the Stanford group. I think we have 20 patients remaining at risk at 1 year, 17 at 2 years, 13 at 3 years, 10 at 4 years, 6 at 5 years, and 2 at 6 years.

Dr Miller. That is the safe way. We did that the same in our 2005 paper presented in Victoria, which Dr Cohn hopefully will be publishing soon in the Journal. (laughter) We had 8 patients at 3 years and 6 patients at 5 years, and you just cannot say anything with such a small number of patients out at the end of the curves.

Dr Khoynezhad. Absolutely.
Dr Miller. A word of caution. In the ruptures you want to cover the entire descending thoracic aorta, and I understand why because you have no idea where they are ruptured, but you only have 4 ruptures. If you do that in enough patients, you are going to pay a price, and the price can be paraplegia. I think we should be cautious. It reminds me of Frank Criado, a vascular surgeon where Ali got his training at Torrance, saying to cover it all with impunity. That does not work out in the patient's best interest all the time. I would have to disagree with you there, even though you are flying blind. You do not know where the actual leak is.

I also have an affirmation. You make 2 pleas in your manuscript. You say that we have to categorize these patients strictly. I could not agree more because there is a lot of fuzziness in how these patients are presented. Second, we have to have uniform standardized outcome-reporting guidelines. It was very heartwarming to me that you are using the Stanford treatment failure definition, which is an overestimate, very comprehensive and too high, but at least it will be a level playing field. We can apply it from center to center and make these comparisons in a more meaningful fashion.

Your $82 \%$ freedom from treatment failure at 5 years is spectacularly good. Our number was $67 \%$ at 5 years.

There are members in the audience who might say your $30 \%$ endoleaks is bad, and this represents a failure of therapy. That is not at all correct. These are acute dissections. Our goal is not to get all the blood flow out of the false lumen. Our goal is to save lives, and that means to pop open the true lumen, improve the malperfusion, resuscitate the patient, and then maybe deal with the persistently patent false lumen another way when they are in much better condition. Therefore I do not interpret that as failure in this context, and I applaud you for pointing that out to the readers.

Dr Khoynezhad. I think endovascular treatment for complicated type $\mathrm{B}$ aortic dissection might not be curative for many patients, but it saves lives in the acute phase, and we always have options to treat patients with open or endovascular means in the future.

Dr Miller. The definition should be applied across the board, absolutely. It is the only way to be fair.

Finally, I applaud what you are doing in Omaha at the University of Nebraska. You are a cardiothoracic surgeon. You did your endovascular training with peripheral vascular surgeons. That represents a failure of our CT training structure in this country right now. Hopefully it will improve in the future, but you are outgrowing those bad habits you might have learned in Torrance, and you are doing what is best for the patient in Omaha. Congratulations.

Dr Royce Calhoun (Sacramento, Calif). Ali, I enjoyed your talk as well. As you know, we stent a lot of traumatic patients, which I think actually is another subset that benefits from this technology, although Dr Miller, I am sure, will take some issue with that. Nonetheless, we see a lot of these patients too, and we have not been as aggressive stenting them. I think the whole pain and uncontrolled hypertension thing is such a vague surgeon-dependent judgment. In at least the ones that we deal with, and we deal with probably 20 to 30 of these a year, that really represents-First of all, they are mostly not complicated. They are mostly uncomplicated once we get their blood pressure down. Of the ones that are complicated, usually it is sort of vague pain, waxing and waning, and hypertension. Of your group, and I realize most of this probably represents Harbor's data, do you know how many of those were truly a sort of malperfusion or 
something where you really had to act versus a judgment about blood pressure management or something like that?

Dr Khoynezhad. Fifteen patients had malperfusion; 8 had malperfusion of the lower extremities, and 7 had visceral/renal malperfusion. As you mentioned, uncontrolled hypertension or pain is a soft call for this procedure. This was the case in 6 patients. That is one of the points in this article, if Dr Cohn agrees to publish it. (laughter) The point is basically that we have to define maximal medical therapy. At my institution, I have established a protocol: 3 parenteral antihypertensive medications have to be initiated before we call that case a failure of medical treatment. This has not been addressed in a majority of articles that I see in the literature currently on complicated acute type B dissections. Having said that, operating on patients with unrelenting pain or incontrollable hypertension has been the standard of care for open surgical repair. Therefore it is fair to treat those patients also endovascularly. These patients who continue to have pain are operated on in aortic centers of excellence, such as the one in Houston with Dr Safi, with whom I have had the privilege and pleasure to work early in my career. 